\title{
Microvascular Obstruction: The Bane of Myocardial Reperfusion
}

\author{
Heerajnarain Bulluck ${ }^{\mathrm{a}, \mathrm{b}}$ and Derek J Hausenloy ${ }^{\mathrm{a}, \mathrm{b}, \mathrm{c}, \mathrm{d}, *}$ \\ ${ }^{a}$ The Hatter Cardiovascular Institute, University College London, London, United Kingdom
}

${ }^{b}$ The National Institute of Health Biomedical Research Centre, University College London Hospitals, London, United Kingdom

'National Heart Research Institute Singapore, National Heart Centre Singapore, Singapore

${ }^{d}$ Cardiovascular and Metabolic Disorders Program, Duke-National University of Singapore, Singapore

* Corresponding author: Cardiovascular and Metabolic Disorders Program, DukeNational University of Singapore, 8 College Road, Singapore 169857, Singapore.

E-mail address: derek.hausenloy@duke-nus.edu.sg (D. Hausenloy)

Tel +65 66015121/65166719 
The field of interventional cardiology has made significant progress over the past three decades in the management of patients with ST-segment elevation myocardial infarction (STEMI), with the introduction of thrombolysis and primary percutaneous coronary intervention as the mainstay treatment. Consequently, this has led to a significant reduction in morbidity and mortality in these patients. ${ }^{1}$ However, despite these advances, in-hospital mortality remains high at approximately $5-6 \%$, increasing to $7-18 \%$ at one year. ${ }^{2}$ This is partly due to the myocardial injury and cardiomyocyte death, which paradoxically occurs despite achieving patency of the infarct-related epicardial coronary artery - termed 'myocardial reperfusion injury'. Four types of myocardial reperfusion injury have been described, namely reperfusion-induced arrhythmias, myocardial stunning, microvascular obstruction (MVO) and lethal myocardial reperfusion injury. ${ }^{3}$ The former two are selflimiting and reversible. The latter two are irreversible and induce cardiomyocyte death and have been the focus of intense research over the past three decades. Crucially, there currently exists no effective therapy for preventing these lethal forms of myocardial reperfusion injury.

MVO, which manifests clinically as coronary no-reflow in the infarct-related artery following primary percutaneous coronary intervention, has been defined as the "inability to reperfuse a previously ischaemic region". ${ }^{4}$ In a recent meta-analysis using patient-level data of more than 1000 patients, MVO by cardiovascular magnetic resonance imaging was found to be present in $54.9 \%$ of primary percutaneous coronary intervention patients despite the presence of normal coronary flow within the infarct-related artery postprimary percutaneous coronary intervention. ${ }^{5}$ This study concluded that MVO was an independent predictor of major adverse cardiovascular events and cardiac death, whereas infarct size as a percentage of the left ventricle was not independently associated with 
major adverse cardiovascular events. ${ }^{5}$ Therefore, there is a pressing need to investigate MVO as a therapeutic target for studies targeting reperfusion injury in STEMI patients.

The animal model of acute myocardial ischemia-reperfusion injury remains a fundamental research tool necessary for the translation of basic science knowledge into the clinical setting. The porcine heart has similar anatomic and haemodynamic resemblance to the human heart ${ }^{6}$ and therefore has become the preferred model in cardiovascular research.

In the article published in Revista Española de Cardiologia, Hervas et $\mathrm{al}^{7}$ investigate the dynamics of MVO in the porcine heart using intracoronary Thioflavin-S (T-S). The main objectives of their study were 2 -fold. Firstly, they wanted to provide evidence for the prerequisite of acute ischemia-reperfusion injury for the development of MVO and its temporal evolution. Secondly, they aimed to compare the intra-coronary administration of T-S against two other conventional routes of administration of T-S (intra-ventricular and intra-aortic) to visual MVO. They used a porcine model of acute myocardial infarction by inflating an angioplasty balloon in the mid-left anterior descending coronary artery for 90 minutes followed by reperfusion. Intra-coronary T-S solution was selectively infused into the proximal left anterior descending through a catheter at 1-minute, 1-week, and 1-month post-reperfusion. In the no-reperfusion group, an over-the-wire balloon was used and was left inflated after 90 minutes. The T-S was then injected through the lumen of the overthe-wire balloon. In the control group, the angioplasty balloon was not inflated. Hearts were then arrested and excised for histological evaluation (visualization of T-S staining under fluorescent light for MVO and staining with 2,3,5-triphenyltetrazolium chloride for infarct size). The second part of their experiment was intra-aortic and intra-ventricular injection of T-S at one week following the same ischemia-reperfusion protocol. The main findings of their study were: (1) MVO, defined as absence of T-S stain, occurred in all the 
reperfused hearts and as early as 1 minute after reperfusion, whereas none of the hearts in the non-reperfused group had MVO. The extent of MVO was largest in the 1-week reperfusion group and smallest in the 1-month reperfusion group; (2) the signal intensity ratio between the areas of MVO and non-MVO in the reperfused territory was higher in the intra-coronary group compared to the intra-aortic and intra-ventricular groups, providing better differentiation of MVO. The authors concluded that myocardial reperfusion plays a critical role in the development of MVO and hence the importance of this critical window in STEMI patients. Secondly, the intra-coronary route for T-S administration allowed accurate characterization of MVO when compared to the conventional intra-aortic and intra-ventricular routes of administration.

Hervas et $\mathrm{al}^{7}$ should be congratulated for their study which provides interesting insights into the pathophysiology of MVO in the reperfused heart. Firstly, their study reinforces the fact that MVO is a consequence of myocardial reperfusion injury and therefore any future studies targeting the reduction of MVO should be initiated prior to reperfusion of the infarct-related artery in order to gain maximum cardioprotection. Khan et al ${ }^{8}$ recently reported in a cohort of 94 STEMI patients (21 presented late and had no reperfusion therapy) that MVO by cardiovascular magnetic resonance imaging was not exclusive to reperfusion therapy and was primarily related to duration of ischemia. However, coronary angiography was performed in only 15 of those patients and 2 patients had Thrombolysis in Myocardial Infarction (TIMI) flow 2 and 4 patients had TIMI flow 1 on the diagnostic images. It is highly likely that some of these patients already had intermittent reperfusion and ischemia and given the significant number of patients having some form of spontaneous reperfusion with TIMI flow $>0$, this would explain the occurrence of MVO in these patients. 
T-S is a fluorescent dye used to assess the patency of the microvascular circulation by staining endothelial cells in patent vessels during reperfusion. ${ }^{9}$ Although Hervas et $\mathrm{al}^{7}$ showed that MVO by T-S staining occur as early as one minute after reperfusion, their study would have been more robust if they were able to use a second parameter such as carbon black and microsphere ${ }^{10}$ or electron microscopy of the microvasculature and tissue characterization by cardiac magnetic resonance imaging to further characterize the areas of MVO.

Hervas et $\mathrm{al}^{7}$ also attempted to provide some insight into the temporal evolution of MVO at 1 minute, 1 week and 1 month post reperfusion. Rochitte et $\mathrm{al}^{11}$ had previously shown that the extent of MVO increased significantly over the first 48 hours following reperfusion in a canine model. Orn et $\mathrm{al}^{12}$ have shown a higher prevalence of MVO by cardiovascular magnetic resonance imaging at 2 days than at 1 week in STEMI patients. Furthermore, a recent abstract by Carrick et $\mathrm{a}^{13}$ reported on serial scanning in 30 STEMI patients at 4 time points (4-12 hours, 3 days, 10 days and 6-7 months post reperfusion). The amount of MVO was greatest at 4-12 hours and fell progressively over time and the amount of intramyocardial hemorrhage increased from 4-12 hours and peaked 3 days. Therefore, the observation from this study that MVO reached its largest extent at 1 week is not accurate and additional time-points between 1-minute and 1-week would have provided a more accurate evolution of MVO.

T-S has always been predominantly administered via the intravenous, intra-atrial, intraaortic or intra-ventricular route. However, the same group has already previously used intracoronary T-S administration in previous studies ${ }^{14,15}$ but in this study provided direct comparison of the signal intensities for MVO detection when compared to the intra-aortic and intra-ventricular routes of administration. The intra-coronary route offered better 
definition between MVO and the reperfused tissue and this will no doubt be a valuable technique for future research studies on MVO.

Despite its limitations, this study by Hervas et $\mathrm{al}^{7}$ provides a platform for future research in this field. Although we know that MVO portends to poor prognosis, ${ }^{5}$ little is known about the ideal timing for MVO imaging and the extent of MVO which best predicts outcome, and more work needs to be done on developing strategies to minimize MVO and translating them to clinical practice to eventually improve outcome in these patients.

\section{CONFLICTS OF INTEREST}

None declared.

\section{REFERENCES}

1. Fox KA, Steg PG, Eagle KA, Goodman SG, Anderson FA, Jr., Granger CB, et al. Decline in rates of death and heart failure in acute coronary syndromes, 1999-2006. JAMA. 2007;297:1892-900.

2. O'Gara PT, Kushner FG, Ascheim DD, Casey DE, Jr., Chung MK, de Lemos JA, et al. 2013 ACCF/AHA guideline for the management of ST-elevation myocardial infarction: executive summary: a report of the American College of Cardiology Foundation/American Heart Association Task Force on Practice Guidelines. Circulation. 2013;127:529-55.

3. Yellon DM, Hausenloy DJ. Myocardial reperfusion injury. N Engl J Med. 2007;357:1121-35.

4. Krug A, Du Mesnil de R, Korb G. Blood supply of the myocardium after temporary coronary occlusion. Circulation Res. 1966;19:57-62. 
5. van Kranenburg M, Magro M, Thiele H, de Waha S, Eitel I, Cochet A, et al. Prognostic value of microvascular obstruction and infarct size, as measured by CMR in STEMI patients. JACC Cardiovasc Imaging. 2014;7:930-9.

6. Lelovas PP, Kostomitsopoulos NG, Xanthos TT. J Am Assoc Lab Anim Sci. 2014;53:432-8.

7. Hervas A, et al. REC-D-15-00062R1. CROSS-REFERENCE.

8. Khan JN, Razvi N, Nazir SA, Singh A, Masca NG, Gershlick AH, et al. Prevalence and extent of infarct and microvascular obstruction following different reperfusion therapies in ST-elevation myocardial infarction. J Cardiovasc Magn Reson. 2014;16:38.

9. Schlegel JU. Demonstration of blood vessels and lymphatics with a fluorescent dye in ultraviolet light. Anat Rec. 1949;105:433-43.

10. Kloner RA. No-reflow phenomenon: maintaining vascular integrity. J Cardiovasc Pharmacol Ther. 2011;16:244-50.

11. Rochitte CE, Lima JA, Bluemke DA, Reeder SB, McVeigh ER, Furuta T, Becker LC, Melin JA: Magnitude and time course of microvascular obstruction and tissue injury after acute myocardial infarction. Circulation 1998, 98(10):10061014.

12. Orn S, Manhenke C, Greve OJ, Larsen AI, Bonarjee VV, Edvardsen T, Dickstein K. Microvascular obstruction is a major determinant of infarct healing and subsequent left ventricular remodelling following primary percutaneous coronary intervention. Eur Heart J. 2009;30:1978-85.

13. Carrick D, Haig C, Ahmed N, Eteiba H, McEntegart M, Watkins S, Lindsay M, Radjenovic A, Oldroyd KG, Berry C: Myocardial haemorrhage after acute reperfused ST-elevation myocardial infarction: temporal evolution, relation 
to microvascular obstruction and prognostic significance. Heart 2015; 101(Suppl 2): A4-A5.

14. Bodi V, Ruiz-Nodar JM, Feliu E, Minana G, Nunez J, Husser O, et al: Effect of ischemic postconditioning on microvascular obstruction in reperfused myocardial infarction. Results of a randomized study in patients and of an experimental model in swine. Int J Cardiol. 2014;175:138-46.

15. Bodi V, Sanchis J, Mainar L, Chorro FJ, Nunez J, Monmeneu JV, et al. Right ventricular involvement in anterior myocardial infarction: a translational approach. Cardiovasc Res. 2010;87:601-8. 